DFTT 25/00

\title{
Thermal operators and cluster topology in q-state Potts Model
}

\author{
M. Caselle ${ }^{a, b}$, F. Gliozzi ${ }^{a, b}$ and S.Necco ${ }^{c}$ \\ ${ }^{a}$ Dipartimento di Fisica Teorica dell'Università di Torino \\ ${ }^{b}$ Istituto Nazionale di Fisica Nucleare, Sezione di Torino \\ via P.Giuria 1, I-10125 Torino, Italy \\ ${ }^{c}$ DESY-IfH Zeuthen, Platanenallee 6, D-15738 Zeuthen, Germany
}

\begin{abstract}
We discuss a new class of identities between correlation functions which arise from a local $\mathbb{Z}_{2}$ invariance of the partition function of the $q$-state Potts model on general graphs or lattices. Their common feature is to relate the thermal operators of the Potts model to some topological properties of the Fortuin-Kasteleyn clusters. In particular it turns out that any even correlation function can be expressed in terms of observables which probe the linking properties of these clusters. This generalises a class of analogous relations recently found in the Ising model.
\end{abstract}




\section{Introduction}

One of the simplest and more studied models of statistical mechanics is the $q$-state Potts model [1, 2], which is the basic system symmetric under the permutation group of $q$ elements. It can be defined on any connected graph $G$ associating with each vertex $i=1,2, \ldots, v(G)$ the spin variable $\sigma_{i}=1,2, \ldots, q$. Its partition function at temperature $T=1 / \beta$ is taken to be

$$
Z_{G}=\sum_{\{\sigma\}} e^{-\beta \mathcal{H}}
$$

where $\mathcal{H}=-\sum_{\langle i j\rangle} \delta_{\sigma_{i} \sigma_{j}}$ with $\langle i j\rangle$ ranging over pairs of adjacent vertices in $G$.

A long-standing problem is how to characterise geometrically in this kind of models the fluctuations near a critical point. Contrary to the naive expectation, the clusters made of adjacent sites with aligned spins do not play an important role in this respect. A different definition of cluster was proposed for the Ising model [3] and generalised to the $q$-state Potts model 沺. These clusters are defined as adjacent sites with the same spin connected by bonds with probability $p=1-e^{-\beta}$. Within such a definition, these clusters behave correctly at the critical point, in the sense that their radius and the density of the percolating cluster scale with the correct critical exponents.

The partition function (11) can be rewritten in terms of these clusters using the Fortuin Kasteleyn representation: [5, 6]

$$
Z_{G}(q, v)=\sum_{G^{\prime} \subseteq G} v^{e\left(G^{\prime}\right)} q^{k\left(G^{\prime}\right)},
$$

where $v=\frac{p}{1-p}=e^{\beta}-1$; the summation is over all spanning subgraphs of $G$, namely the subgraphs made with all the vertices of $G$ i.e. with $v\left(G^{\prime}\right)=$ $v(G) ; e\left(G^{\prime}\right)$ is the number of edges of $G^{\prime}$, called active bonds, and $k\left(G^{\prime}\right)$ the number of connected components or Fortuin-Kasteleyn (FK) clusters. This formulation of the partition function, sometimes called a di-chromatic polynomial, enables one to generalise $q$ from positive integers to real and complex values. In particular $q=0$ corresponds to the tree percolation problem and $q=1$ is the random percolation problem.

In the present work we establish some new exact topological properties of these clusters. They arise from a class of identities between correlation functions which are valid for any graph $G$ and come from the invariance of the partition function (11) with respect to a local $\mathbb{Z}_{2}$ transformation. Their 
origin suggests to call them Ward identities (see Sect. 2). Their common feature is to relate the thermal operators of the Potts model to some linking properties of the FK clusters and generalise a class of analogous relations recently found in the Ising model []].

It turns out that any even correlation function can be expressed in terms of a new kind of observables, which depend only on the closed circuits of these clusters (see Sect. 3). This suggests new, powerful methods to evaluate even correlators which can be easily implemented in numerical experiments: let $G_{1}^{\prime}, G_{2}^{\prime}, \ldots, G_{i}^{\prime}, \ldots$ be a sequence of spanning subgraphs in statistical equilibrium with the partition function (2), generated by any updating algorithm. Deleting all the edges which do not belong to any circuit of $G_{i}^{\prime}$ produces a dramatic simplification of the configuration with no information loss on thermal properties of the system. In the simplified configurations the evaluation of the new kind of observables is a much easier task. This leads to improved estimators of the thermal operators of this model.

\section{Ward Identities}

An elementary derivation of the simplest of these identities is the following. Let $\ell$ be any edge of $G$. Denote by $G_{\ell}^{+}$the spanning subgraphs containing $\ell$ and by $G_{\ell}^{-}$the others. Clearly the set $\left\{G^{\prime}\right\}$ of all the spanning subgraphs may be written as the sum of two disjoint subsets $\left\{G^{\prime}\right\}=\left\{G_{\ell}^{+}\right\} \cup\left\{G_{\ell}^{-}\right\}$. Denote by $\delta_{\ell}$ the $\mathbb{Z}_{2}$ transformation on $Z_{G}(q, v)$ which deletes the edge $\ell$ from any subgraph of type $G_{\ell}^{+}$and adds it to any subgraph of type $G_{\ell}^{-}$. This is a symmetry of the partition function, namely,

$$
\delta_{\ell}\left[Z_{G}(q, v)\right]=Z_{G}(q, v),
$$

since $\sum_{G^{\prime} \subseteq G}=\sum_{G_{\ell}^{+} \subseteq G}+\sum_{G_{\ell}^{-} \subseteq G}$ and $\delta_{\ell}$ exchanges the two subsets $\left\{G_{\ell}^{+}\right\} \leftrightarrow$ $\left\{G_{\ell}^{-}\right\}$. On the contrary the two partial sums are not invariant, but their way of transforming under $\delta_{\ell}$ can be explicitly evaluated. We have, of course,

$$
\delta_{\ell}\left[e\left(G_{\ell}^{ \pm}\right)\right]=e\left(G_{\ell}^{ \pm}\right) \mp 1 .
$$

The action of $\delta_{\ell}$ on the number of clusters $k\left(G_{\ell}^{+}\right)$depends on a topological property of $\ell$ : if it belongs to a circuit of $G_{\ell}^{+}, k$ is kept invariant

$$
\delta_{\ell}\left[k\left(G_{\ell}^{+}\right)\right]=k\left(G_{\ell}^{+}\right) ;
$$

we call black bonds the edges with this property [7]. If $\ell$ do not belongs to any closed circuit of $G_{\ell}^{+}$we have

$$
\delta_{\ell}\left[k\left(G_{\ell}^{+}\right)\right]=k\left(G_{\ell}^{+}\right)+1
$$


and call it grey bond or bridge. Using (4), (5) and (6) we obtain 1

$$
\delta_{\ell}\left[\sum_{G_{\ell}^{+} \subseteq G} v^{e\left(G_{\ell}^{+}\right)} q^{k\left(G_{\ell}^{+}\right)}\right]=\sum_{G_{\ell}^{+} \subseteq G}\left[\frac{1}{v} \pi_{G_{\ell}^{+}}(\ell)+\frac{q}{v}\left(1-\pi_{G_{\ell}^{+}}(\ell)\right)\right] v^{e\left(G_{\ell}^{+}\right)} q^{k\left(G_{\ell}^{+}\right)}
$$

where $\pi_{G^{\prime}}(\ell)$ denotes a projector on the black bonds defined as follows

$$
\pi_{G^{\prime}}(\ell)= \begin{cases}1 & \text { if } \ell \text { is a black bond } \\ 0 & \text { otherwise }\end{cases}
$$

For the other partial sum we can write, more simply,

$$
\delta_{\ell}\left[\sum_{G_{\ell}^{-} \subseteq G} v^{e\left(G_{\ell}^{-}\right)} q^{k\left(G_{\ell}^{-}\right)}\right]=\sum_{G_{\ell}^{+} \subseteq G} v^{e\left(G_{\ell}^{+}\right)} q^{k\left(G_{\ell}^{+}\right)} .
$$

From (3), (7) and (9) one obtains immediately

$$
1=\frac{v+q}{v}\langle\rho(\ell)\rangle-\frac{q-1}{v}\langle\pi(\ell)\rangle,
$$

where $\langle\rho(\ell)\rangle=\sum_{G_{\ell}^{+}} P\left(G_{\ell}^{+}\right)$, with $P\left(G^{\prime}\right)=v^{e\left(G^{\prime}\right)} q^{k\left(G^{\prime}\right)} / Z_{G}(q, v)$.

Summing over all the edges $\ell=1,2 \ldots n \equiv e(G)$ yields

$$
n=\frac{v+q}{v}\langle e\rangle-\frac{q-1}{v}\langle b\rangle,
$$

where $\langle e\rangle=\sum_{G^{\prime}} e\left(G^{\prime}\right) P\left(G^{\prime}\right)$ is the mean number of edges of $G^{\prime}$, called active bonds and similarly $\langle b\rangle=\sum_{G^{\prime}} b\left(G^{\prime}\right) P\left(G^{\prime}\right)$, where $b\left(G^{\prime}\right)$ is the number of black bonds in the subgraph $G^{\prime}$. This is the first Ward identity. Since $\langle e\rangle$ is related to the internal energy $E$ according to

$$
E \equiv-\frac{\partial \log Z_{G}}{\partial \beta}=-\frac{v+1}{v}\langle e\rangle
$$

we can rewrite the above identity in the form

$$
-E=\frac{v+1}{v+q} e(G)+\frac{(q-1)(v+1)}{v(v+q)}\langle b\rangle,
$$

\footnotetext{
${ }^{1}$ This way of reasoning is very similar to the one used to describe the basic properties of the Tutte polynomial of a graph [8], which is a simple variant of the partition function (2); see also [9].
} 
which gives the internal energy $E$ in terms of black bonds. This illustrates a common feature of this kind of Ward identities: all the thermal properties of the model are encoded in the black bond configurations, as we shall see shortly. The fact that the energy counts this special kind of bonds has been already observed some time ago [11].

As a little diversion, let us remark that if $G$ were a tree $T$, then $b(T) \equiv$ $b\left(G^{\prime}\right) \equiv 0$. This leads to $E=-\frac{v+1}{v+q} e(T)$, which is a well known result of the $q$-state Potts model on any tree with sub-exponential growth [10]; it also holds in the symmetric phase of any Bethe lattice .

It is also interesting to study Eq. (11) in the pure percolation limit $q \rightarrow 1$. Taking this limit together with the Taylor expansion of $\langle e\rangle$

$$
\langle e\rangle=\frac{v e(G)}{v+1}+(q-1)[\langle e k\rangle-\langle e\rangle\langle k\rangle]_{q=1}+\ldots,
$$

yields the curious identity

$$
\frac{\langle e-b\rangle_{q=1}}{v+1}=[\langle e\rangle\langle k\rangle-\langle e k\rangle]_{q=1}
$$

relating the mean number of bridges to the connected correlator between edges and clusters.

Other Ward identities can be simply obtained by applying the above $\mathbb{Z}_{2}$ transformation to more than one edge. In particular, the two-edge transformation leads to the following expression of the specific heat $C=\frac{\partial E}{\partial T}$ in terms of black bonds

$$
\begin{aligned}
\frac{C}{\beta^{2}}+E+\frac{E^{2}}{e(G)} & =\frac{(q-1)(v+1)^{2}}{v(v+q)}\{2\langle h\rangle+ \\
& \left.+(q-1)\left[\left\langle b^{2}\right\rangle-\langle b\rangle^{2}-\frac{\langle b\rangle}{n}(n-\langle b\rangle)\right]\right\}
\end{aligned}
$$

with $\langle h\rangle=\sum_{G^{\prime}} h\left(G^{\prime}\right) P\left(G^{\prime}\right)$, where $h\left(G^{\prime}\right)$ denotes the number of the cutting pairs of the spanning subgraph $G^{\prime}$, i.e. the pairs of black bonds such that their deletion increases the number of clusters.

\section{Cutting the bridges}

We come now to the other purpose of this paper, namely to show that not only the internal energy or the specific heat, but also any correlation function involving an even number of sites my be evaluated in terms of black bond 
configurations $B\left(G^{\prime}\right)$, i.e. the graphs generated by deletion of all the bridges in the spanning subgraphs. To this end it is convenient to slightly generalise the model in the following way: we assign to any oriented edge $\langle i j\rangle$ of $G$ a twist, namely an integer $\tau_{i j}=0, \pm 1, \ldots, \pm(q-1)$ and define a modified Hamiltonian

$$
\mathcal{H}_{\tau}=-\sum_{\langle i j\rangle} \delta_{\sigma_{i}, \sigma_{j}+\tau_{i j}}
$$

where the sum $\sigma_{i}+\tau_{i j}$ is taken modulo $q$. An edge $\langle i j\rangle$ with $\tau_{i j} \neq 0$ is said to be twisted. In order to get the Fortuin Kasteleyn representation of the twisted partition function $Z_{G, \tau}=\sum_{\{\sigma\}} \mathrm{e}^{-\beta \mathcal{H}_{\tau}}$, we can rewrite it as

$$
Z_{G, \tau}=\sum_{\{\sigma\}} \prod_{\langle i j\rangle}\left(1+v \delta_{\sigma_{i}, \sigma_{j}+\tau_{i j}}\right), \quad\left(v=\mathrm{e}^{\beta}-1\right)
$$

and expand the product as a sum of terms corresponding to the possible choices of summand from each factor. Each edge of the graph $G$ can appear with a factor 1 or $v \delta_{\sigma_{i}, \sigma_{j}+\tau_{i j}}$; in the latter case the edge is said to be an active bond. In a term with $e\left(G^{\prime}\right)$ active bonds we have a factor $v^{e\left(G^{\prime}\right)}$ together with a product of delta functions which forces to zero every configuration $G^{\prime}$ in which all the spins connected by the active bonds do not satisfy the constraint $\delta=1$. If there are no twists in $G^{\prime}$ this constraint implies that all the spins of each cluster are aligned and the total number of spin configurations in a situation in which $G^{\prime}$ is formed by $k\left(G^{\prime}\right)$ clusters is $q^{k\left(G^{\prime}\right)}$. If, on the contrary, there are twisted edges on $G^{\prime}$, we get a non-vanishing contribution only if there are no frustrations, namely if and only if the algebraic sum of the twists along any circuit of $G^{\prime}$ is zero modulo $q$. In order to select these subgraphs let us introduce a projector $\varpi_{\tau}\left(G^{\prime}\right)$ defined as

$$
\varpi_{\tau}\left(G^{\prime}\right)= \begin{cases}1 & \text { if } G^{\prime} \text { has no frustrations } \\ 0 & \text { otherwise. }\end{cases}
$$

Note that this projector picks out a subgraph $G^{\prime}$ regardless the location of its bridges, thus it can only be a function of the black bond configurations $B\left(G^{\prime}\right)$ :

$$
\varpi_{\tau}\left(G^{\prime}\right)=\varpi_{\tau}\left(B\left(G^{\prime}\right)\right) .
$$

In terms of this projector we have

$$
Z_{G, \tau} \equiv \sum_{\{\sigma\}} e^{-\beta \mathcal{H}_{\tau}}=\sum_{G^{\prime} \subseteq G} \varpi_{\tau} v^{e\left(G^{\prime}\right)} q^{k\left(G^{\prime}\right)}
$$


this leads to the fundamental identity

$$
\frac{Z_{G, \tau}}{Z_{G}}=\left\langle\varpi_{\tau}\right\rangle
$$

where the expectation value is taken with respect to the standard, untwisted Hamiltonian. This defines a (complete) set of physical observables, one for each choice of $\tau$, which depend only on the black bond configurations and probe the linking properties of the FK clusters.

Thus the problem reduces to show that any even correlation function can be expressed in terms of suitable combinations of $\varpi_{\tau}$ 's. This can be done, at least in principle, by writing the expectation value (22) in terms of spin variables

$$
\left\langle\varpi_{\tau}\right\rangle=\frac{\sum_{\{\sigma\}} \mathrm{e}^{-\beta \mathcal{H}_{\tau}}}{\sum_{\{\sigma\}} \mathrm{e}^{-\beta \mathcal{H}}}=\left\langle\mathrm{e}^{\beta \sum_{\langle i j\rangle}\left(\delta_{\sigma_{i}, \sigma_{j}+\tau_{i j}}-\delta_{\sigma_{i} \sigma_{j}}\right)}\right\rangle
$$

and then expanding the exponential. As examples, it is easily verified that a single twisted edge generates the first Ward identity (11) and the two-edge twist yields eq.(16).

Note that eq. (23) has the typical form of the expectation value of correlators of disorder observables [12]. Measuring disorder observables is not an easy task: one needs expectation values of operators which are exponentially suppressed on dominant configurations of the statistical ensemble. In particular, the numerical evaluation of operators involving a large number of twisted links represents a very hard sampling problem. Actually Eq. (20) yields a simple, effective way out. Indeed $\varpi_{\tau}\left(B\left(G^{\prime}\right)\right)$ is an improved estimator of the same observable. The evaluation of $\varpi_{\tau}$ on the black bond configurations is much less noisy than in the spin configurations, because in the former case $\varpi_{\tau}$ can only take the values 0 and 1 , while in the latter case the exponential of spin variables has a much larger variance, especially for large distance correlators. As a matter of fact, powerful algorithms to evaluate these new estimators have been constructed for the surface tension in 3D Ising model [13 and for the Wilson loops Polyakov correlators in 3D $\mathbb{Z}_{2}$ gauge model 14 - 15 . Their extension to a general $q-$ state Potts model is now straightforward.

This work has been supported in part by the Ministero italiano dell'Università e della Ricerca Scientifica e Tecnologica.

\section{References}


[1] R.B. Potts, Proc. Camb. Phil. Soc. 48, 106 (1952)

[2] F.Y. Wu, Rev. Mod. Phys. 54, 235 (1982)

[3] A. Coniglio and W. Klein, J. Phys. a 12, 2775 (1980)

[4] A. Coniglio and F. Peruggi, J. Phys. A 15, 1873 (1982)

[5] H. Whitney, Ann. of Math. 38, 572 (1932)

[6] P.W. Kasteleyn and C.M. Fortuin, J. Phys. Soc. Jpn. 26 (Suppl.), 11 (1969); C.M. Fortuin and P.W. Kasteleyn, Physica 57, 536 (1972)

[7] M. Caselle and F. Gliozzi, J. Phys. A 33, 2333 (2000)

[8] W.T. Tutte, Canad. J. Math. 6, 80 (1954)

[9] B. Bollobás, Modern Graph Theory, Springer (1998)

[10] R. Burioni and D. Cassi, Mod. Phys. Lett. B 7, 1947 (1993)

[11] J. Cardy, Scaling and Renormalization in Statistical Physics, Cmbridge Lecture Notes in Physics, page 160 (1997)

[12] L.P. Kadanoff and H. Ceva, Phys. Rev. B 3, 3918 (1971)

[13] M. Hasenbusch, J.Phys. I 3, 753 (1993)

[14] M. Caselle, R. Fiore, F. Gliozzi, M. Hasenbusch and P. Provero P, Nucl. Phys. 486, 245 (1997)

[15] F. Gliozzi and P. Provero, Phys. Rev. D56 , 1131 (1997); F. Gliozzi and S. Vinti, Nucl. Phys. B 53 (Proc. Suppl.) 593, (1997) 